\title{
Evaluation of In-vitro Anti-Inflammatory Activity of Silver Nanoparticles Synthesised using Piper Nigrum Extract
}

\section{Aparna Mani KM ${ }^{1 *}$, Seethalakshmi $\mathbf{S}^{1}$ and Gopal $\mathbf{V}^{\mathbf{2}}$}

${ }^{1}$ Department of Pharmacology, Sri Ramachandra Medical College \& Research Institute, Chennai, Tamil Nadu, India

${ }^{2}$ Department of Pharmacognosy, College of Pharmacy, Mother Theresa Post Graduate and Research Institute of Health Sciences, Puducherry, India

\begin{abstract}
Introduction: Nanotechnology has emerged as an exciting approach in the drug development process and among the various nanoparticles, silver nanoparticles have been explored for its variety of medical applications. Phyto assisted synthesis of silver nanoparticles is an eco-friendly and cost effective method for the development of silver nanoparticles with additional properties conferred by the capping phytochemicals.
\end{abstract}

Aim of the study: To synthesize silver nanoparticles using the aqueous extract of the unripe fruits of Piper nigrum and to evaluate its in-vitro anti inflammatory activity.

Results: The synthesised silver nanoparticles were characterized using UV-Spectroscopic analysis, Scanning Electron Microscopy (SEM), Fourier Transform Infra Red Spectroscopy (FTIR) analysis, Atomic Absorption Spectroscopy (AAS) and High Performance Thin Layer Chromatography (HPTLC). The alkaloids and proteins present in Piper nigrum extract act as both reducing and capping agents. The synthesised silver nanoparticles were spherical and cuboidal with a size range of $40-100 \mathrm{~nm}$. HPTLC studies revealed that $856 \mathrm{ng}$ of piperine was found capping $1 \mathrm{mg}$ of silver nanoparticles. The anti inflammatory activity of the synthesised silver nanoparticles was assessed using in-vitro assays for Tumour Necrosis Factor $\alpha$ (TNF $\alpha$ ), Interleukins-1 $\beta$ and 6 (IL-1 $\beta$ and IL-6). The synthesised silver nanoparticles were also compared with the commercial silver nanoparticles, synthesised by standard chemical methods in these assays. It was found that the synthesised silver nanoparticles showed greater inhibition of all three cytokines at concentrations ranging from $10-20 \mu \mathrm{g} / \mathrm{ml}$.

Conclusion: The synthesised silver nanoparticles exhibited an enhanced anti inflammatory activity due to the synergistic effect of alkaloids of Piper nigrum extract and the silver ions.

Keywords: Silver nanoparticles; Piper nigrum; Antiinflammatory

\section{Introduction}

Inflammation is the complex response of the immune system to infection and injury that leads to removal of offending factors and restoration of tissue structure and physiological function [1]. The symptoms of inflammation are characterized by pain, heat, redness, swelling and loss of function. It can be classified into two major types either acute or chronic, based on the duration of the inflammatory reaction. Though initiated as a protective phenomenon, loss of regulation of this complex process can lead to the development of various inflammatory disorders.

The current pharmacological management of inflammation is mainly by two groups of drugs- the steroidal anti inflammatory drugs and the non steroidal anti inflammatory agents. However these conventional drugs are associated with numerous side effects that has compelled the need for identification of alternative substances that can resolve inflammation in a way that is homeostatic, modulatory, efficient, and well-tolerated by the body [2]. One such alternative rationale for treatment of inflammatory disorders is Phytomedicine. Purified natural compounds from plants have aided in the synthesis of new generation antiinflammatory drugs with higher therapeutic value and lower toxicity [3]. The major disadvantage of plant based drugs as pointed out by many published papers is the lack of quality in the production, trade and prescription of phytomedicinal products [4].

Another alternative method to develop newer anti inflammatory agents with sustained release and better efficacy is the use of nanotechnology for drug development and delivery. Nanotechnology deals with the synthesis and fabrication of materials at the nanoscale level (1-100 nm) [5]. Among the various nanoparticles available, silver nanoparticles are gaining more importance due to their diversified biological properties and potential applications. Silver has been used since ancient times for the treatment of wounds and inflammation and nanoparticles of silver have been developed which have potent antiinflammatory [6] and antioxidant activities [7].

Silver nanoparticles can be synthesised by various physical and chemical methods, however they are costly, cumbersome and toxic to the environment. The use of biological systems as potential nanofactories has been widely explored as they are economical and ecofriendly. Plant extracts contain phyto-chemicals which aid in the reduction of the silver ions [8]. The added advantage of using plants is that the alkaloids or flavanoids also act as capping agents, thereby conferring the silver nanoparticles with additional pharmacological properties.

Piper nigrum (commonly referred as Black pepper) is a tropical climber belonging to the family Piperaceae and is mainly cultivated for

*Corresponding author: Aparna Mani KM, Senior Resident, Department of Pharmacology, Sri Ramachandra Medical College and Research Institute Porur, Chennai-600116, Tamil Nadu, India, Tel: 91-9095009724; E-mail: draparnacmc@gmail.com

Received October 20, 2014; Accepted January 28, 2015; Published February 08, 2015

Citation: Aparna Mani KM, Seethalakshmi S, Gopal V (2015) Evaluation of In-vitro Anti-Inflammatory Activity of Silver Nanoparticles Synthesised using Piper Nigrum Extract. J Nanomed Nanotechnol 6: 268. doi:10.4172/2157-7439.1000268

Copyright: () 2015 Aparna Mani KM, et al. This is an open-access article distributed under the terms of the Creative Commons Attribution License, which permits unrestricted use, distribution, and reproduction in any medium, provided the original author and source are credited. 
its fruit which in used as a spice and for seasoning. The fruits contain Piperine which is the major alkaloid responsible for its pungency. It has been used in traditional medicine to treat various disease conditions including indigestion, insect bites, joint pains, liver problems, insomnia and toothache. The biological activities of Piper nigrum have been studied extensively and the plant possesses significant analgesic, anti-inflammatory [9], and antioxidant properties [10,11]. Piperine is also known to have bio enhancing properties, and increases the bioavailability of several drugs and compounds [12].

The present study was undertaken to synthesize silver particles using the aqueous extract of the unripe fruits of Piper nigrum and to evaluate the silver nanoparticles for anti- inflammatory activity using standard in-vitro methods. Thus by synthesising silver nanoparticles using Piper nigrum extract, the potential advantages of phytomedicine and nanomedicine can be combined to result in a more enhanced and synergistic anti-inflammatory effect.

\section{Materials and Methods}

\section{Synthesis of silver nanoparticles}

To $100 \mathrm{ml}$ of $1 \mathrm{mM} \mathrm{AgNO}_{3}$ solution, $1 \mathrm{ml}$ of the aqueous extract of unripe fruits of Piper nigrum was added and the mixture was kept in a magnetic stirrer for $2 \mathrm{hrs}$. The mixture was then centrifuged at 3000 $\mathrm{rpm}$ for $10 \mathrm{~min}$ and the residue was used for characterization studies [13]

\section{Characterization of silver nanoparticles}

UV-Visible spectroscopy: The bioreduction of the silver ions in solution was measured after 2 hours of magnetic stirring. A small aliquot of the sample was diluted with distilled water and observed in the wavelength range of 300-500 nm using ELICO Double beamSL210 UV Vis Spectrophotometer.

Scanning Electron Microscopy (SEM) analysis: Scanning electron microscopic analysis was done to determine the morphology of the silver nanoparticles biosynthesized using Piper nigrum extract. SEM analysis was done using HITACHI S 4500-SEM Machine.

Fourier Transform Infrared Spectroscopy (FTIR) analysis: The synthesized silver nanoparticles were subjected to FTIR analysis to determine the functional groups capping the particles. Analysis was done by using $\mathrm{KBr}$ pellet (FTIR grade) method in 1:100 ratios and spectrum was recorded in Nicolet Impact 400 FT-IR Spectrophotometer.

Atomic absorption spectroscopy: This spectroanalytical technique was done to determine the quantity of silver in the synthesized silver nanoparticles. The synthesized silver nanoparticles were subjected to atomic absorption spectroscopy using AA 6200 Shimadzu instrument.

High Performance Thin Layer Chromatography (HPTLC) analysis: HPTLC analysis was done to estimate the quantity of Piperine in the synthesized silver nanoparticles. HPTLC analysis was done using CAMAG Automatic TLC Sampler 4 (ATS4). HPTLC was performed using $10 \mathrm{~cm} \times 20 \mathrm{~cm}$ aluminum backed Silica gel F254 HPTLC plates from E.Merck (Germany) as stationary phase and n-Hexane: ethyl acetate (30:70) as the mobile phase.

\section{In vitro assay for anti-inflammatory activity}

In vitro assay used for evaluating the anti- inflammatory property was LPS induced expression of TNF $\alpha$, IL- $1 \beta$ and IL- 6 in human peripheral blood mononuclear cells (PBMC). The ability of the synthesized silver nanoparticles to inhibit the expression of TNF $\alpha$ and
IL- $1 \beta$ were assessed by RT-PCR and inhibition of IL- 6 expression was evaluated using ELISA kit method.

Chemicals: Dimethyl sulfoxide (DMSO), phosphate-buffered saline (PBS), and LPS were purchased from Sigma Chemicals Co.

Collection of blood sample: $10 \mathrm{ml}$ of peripheral venous blood was collected from a healthy volunteer, with no history of metabolic and infectious diseases and with no history of recent or long term drug intake. Written informed consent was obtained from the participant before the start of the study and the experimental protocol was reviewed and approved by the Institutional Ethics Committee, Sri Ramachandra Medical College and Research Institute, Porur. (Ref:CSP-MED/13/ JUN/07/40).

Preparation of human PBMC cells: PBMCs were isolated from erythrocytes and granulocytes by density gradient centrifugation and cell numbers were determined using a hemocytometer. PBMCs $\left(2 \times 10^{5}\right.$ cells/well $)$ were incubated with various concentration of silver nanoparticles for $4 \mathrm{hrs}$, and then LPS (Lipo-polysaccharide) was added and incubated for $48 \mathrm{~h}$. The supernatant samples were collected and stored at $-20^{\circ} \mathrm{C}$ until use.

Evaluation of IL-6 expression: Production of IL-6 in the PBMC supernatant samples was measured using DuoSet ELISA kit according to manufacturer instructions.

Evaluation of TNF $\alpha$ and IL-1 $\beta$ expression: The expression of TNF $\alpha$ and IL-1 $\beta$ were assessed using RT-PCR method which involves the following steps:

$\checkmark$ Extraction of total cellular RNA: Total cellular RNA was extracted from PBMCs by using Qiagen kit procedure.

$\checkmark$ Determination of mRNA expression by reverse transcriptionpolymerase chain reaction (RT-PCR): Total RNA was reversetranscribed to generate cDNA using high cDNA synthesis RT-PCR kit. The cDNA pool was subjected to quantitative PCR by using SYBR green PCR Master Mix (Applied Biosystems) on the AB 7500 fast real time PCR system (Applied Biosystems). The primers for TNFa, and IL- $1 \beta$ were normalised with house keeping gene $\beta$-actin (Table 1 ). The following conditions were used to quantify the genes: $2 \mathrm{~min}$ at $50^{\circ} \mathrm{C}$, $10 \mathrm{~min}$ at $95^{\circ} \mathrm{C}$, and 40 cycles of $15 \mathrm{~s}$ at $95^{\circ} \mathrm{C}$ and $1 \mathrm{~min}$ at $60^{\circ} \mathrm{C}$. PCR products were verified by melting curve analysis. The changes in the threshold cycle $\left(\mathrm{C}_{\mathrm{T}}\right)$ values were calculated by the equation- $\mathrm{C}_{\mathrm{T}}=\mathrm{C}_{\mathrm{T} \text { (test) }}$ $-\mathrm{C}_{\mathrm{T}}$ (control) and the fold difference was calculated as: fold difference= 2-(CT) Data presented as the mean \pm SEM from triplicate experiments.

\section{Statistical analysis}

Statistical analysis for the in vitro anti inflammatory assays was performed using IBM SPSS version 19.0. Data was expressed as the mean \pm SEM. The data were analyzed using one way analysis of variance (ANOVA) followed by Post hoc-Tukey's test. Data were considered significant if the $\mathrm{p}$ values were below $0.05(\mathrm{p}<0.05)$.

\section{Results}

In the present study, silver nanoparticles were synthesized using Piper nigrum extract and evaluated for anti inflammatory property.

\begin{tabular}{|l|l|l|}
\hline Gene & Primer Sequence: Forward & Primer sequence: $R$ Reverse \\
\hline TNF a & 5'CGAGTGACAAGCCTGTAGCC3' & 5TTGAAGAGGACCTGGGAGTAG3' \\
\hline IL-1 $\beta$ & 5'CTCTCTCACCTCTCCTACTCAC3' $^{\prime}$ & 5'ACACTGCTACTTCTTGCCCC3' \\
\hline$\beta$-actin & 5'AACTGGAACGGTGAAGGTG3' & 5'CTGTGTGGACTTGGGAGAGG3' \\
\hline
\end{tabular}
Table 1: Primers for RTPCR [14]. 
Silver nanoparticles using Piper nigrum extract were synthesized by magnetic stirring method within $2 \mathrm{hrs}$ of reaction time. The reduction of silver ions to silver nanoparticles by the aqueous extract of Piper nigrum was evidenced by the darkening of the brown colour of the extract after adding $1 \mathrm{mM}$ silver nitrate solution. This change in colour was due to the excitation of the surface plasmon vibrations.

\section{Mechanism of silver nanoparticle synthesis}

Piper nigrum aqueous extract is known to contain several biomolecules such as alkaloids, proteins, polysaccharides, amino acids and vitamins. The proposed mechanism for synthesis of silver nanoaparticles is attributed to Piperine, the major alkaloid. The silver ions get trapped on the surface of alkaloid piperine and are then reduced by the proteins leading to the formation of silver nuclei. These formed silver nuclei accumulate and subsequently grow in size resulting in the formation of the silver nanoparticles. The silver nanoparticles are further capped by the alkaloids to prevent their aggregation and also to stabilize them.

\section{Characterisation of synthesised silver nanoparticles}

UV-Visible spectroscopy: The UV visible spectra showed an absorption band at $420 \mathrm{~nm}$ which was specific for the formed silver nanoparticles. The peak was observed after 2 hours of reaction time and the absorption band observed was broad indicating the polydispersity of the nanoparticles. The UV-Visible spectrum of the silver nanoparticles synthesized using Piper nigrum extract is depicted in Figure 1.

SEM analysis: The SEM picture of the synthesized silver nanoparticles is shown in Figure 2. From the picture it can be seen

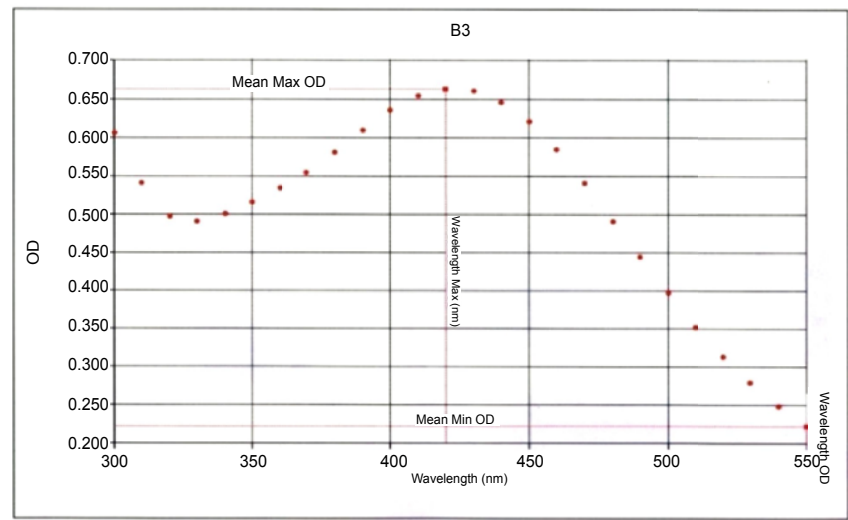

Figure 1: UV-Visible Spectra of Silver nanoparticles synthesized using Pipe nigrum extract.

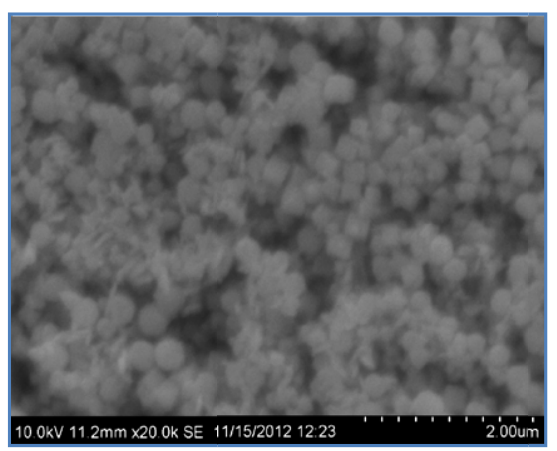

Figure 2: SEM picture of Silver nanoparticles synthesized using Piper nigrum extract. that the synthesized silver nanoparticles were spherical and cuboidal in shape and were well dispersed without any aggregation. The size range of the nanoparticles as assessed by SEM analysis was between 40-100 $\mathrm{nm}$ in size. Such variation in size and shape are common when employing biological systems for the synthesis.

Atomic absorption spectroscopy: The amount of elemental silver in the synthesized silver nanoparticles was determined using atomic absorption spectroscopy and the quantity of silver in 1 gram of synthesised silver nanoparticles was estimated to be $749.2 \mathrm{mg}$.

FTIR analysis: The FTIR spectrum to determine the various functional groups which acted as capping agents is depicted in Figure 3. The synthesized silver nanoparticles exhibited strong absorption peak at $3383 \mathrm{~cm}^{-1}$, corresponding to the $\mathrm{N}-\mathrm{H}$ stretching of primary amine. The bands observed at $1649 \mathrm{~cm}^{-1}$ and $1552 \mathrm{~cm}^{-1}$ indicate the $\mathrm{C}=\mathrm{O}$ stretching of ketones. The peaks observed at $1457 \mathrm{~cm}^{-1}, 1432 \mathrm{~cm}^{-1}$ represent the $\mathrm{N}=\mathrm{O}$ stretching of the nitro groups present in the fruit extract. The smaller peaks at $676 \mathrm{~cm}^{-1}, 642 \mathrm{~cm}^{-1}$ and $828 \mathrm{~cm}^{-1}$ indicate the bending of alkynes. From the FTIR analysis it is evident that the silver nanoparticles are capped with the phytochemicals with various functional groups which give characteristic peaks in the spectrum.

HPTLC analysis: Piperine is the major active ingredient present in Piper nigrum fruits and is also the major alkaloid acting as a capping agent in the biosynthesis of silver nanoparticles (Figure 4). It was found that $1 \mathrm{mg}$ of biosynthesized silver nanoparticles contain $856 \mathrm{ng}$ of piperine.

\section{In vitro anti-inflammatory assays}

Assay for IL-1ß: The effect of synthesized silver nanoparticles (SNP) in inhibiting the expression of IL-1 $\beta$ was assessed and compared with commercially available silver nanoparticle dispersion (CNP). Dexamethasone was used as the standard. The effect of the tests compounds on the mean normalized expression of IL- $1 \beta$ is demonstrated in Figure 5. It can be seen that the biosynthesized silver nanoparticles show potent inhibition of IL-1 $\beta$ expression. Even at lower concentration of $25 \mu \mathrm{g}$, the biosynthesized silver nanoparticles (SNP) show significant inhibition compared to the commercial silver nanoparticle dispersion $(\mathrm{p}<0.01)$.

Assay for TNFa: The effect of synthesized silver nanoparticles in inhibiting TNF $\alpha$ is depicted in Figure 6. It can be seen that at $10 \mu \mathrm{g}$ concentration, the expression of TNFa was significantly inhibited, however it was statistically not significant when compared to the commercial silver nanoparticles (CNP).

Assay for IL-6: Among the various pro-inflammatory cytokines IL-6 plays a major role in the amplification loop of the inflammatory response. The inhibition of IL-6 by various concentrations of synthesized silver nanoparticles is depicted in Figure 7. The synthesized silver nanoparticles inhibit IL-6 expression significantly even at very low concentration of $10 \mu \mathrm{g}$ when compared to commercial silver nanoparticles $(\mathrm{p}<0.05)$.

\section{Discussion}

The use of nano-herbal-technology to synthesize compounds with improved anti inflammatory properties is an area of current research by many scientists. In our study, we report the non toxic, practical and environmentally benevolent approach for the synthesis of silver nanoparticles using the aqueous fruit extract of Piper nigrum plant with potent anti inflammatory activity. 


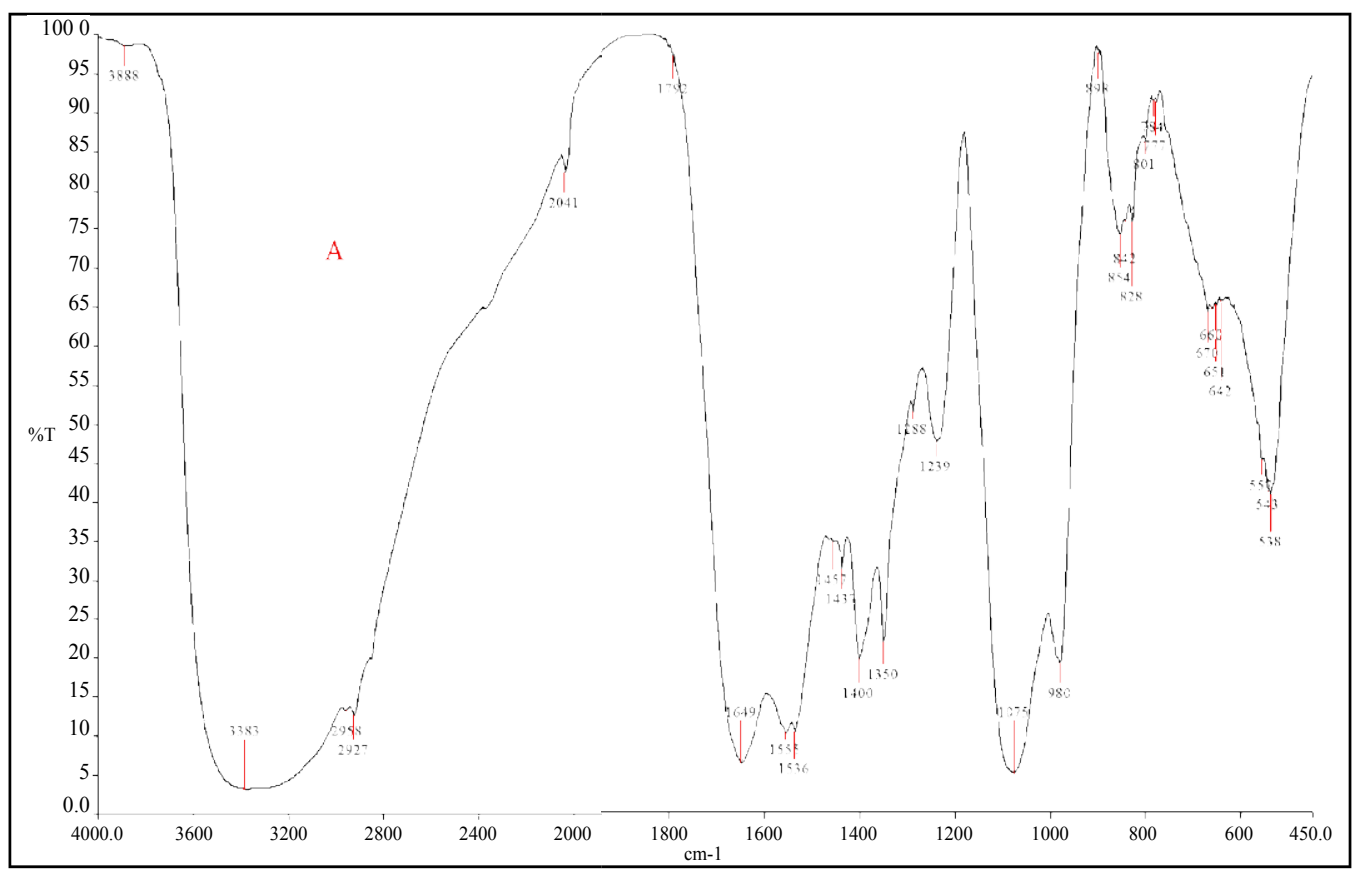

Figure 3: FTIR Spectra of Silver nanoparticles Piper nigrum synthesized using extract.

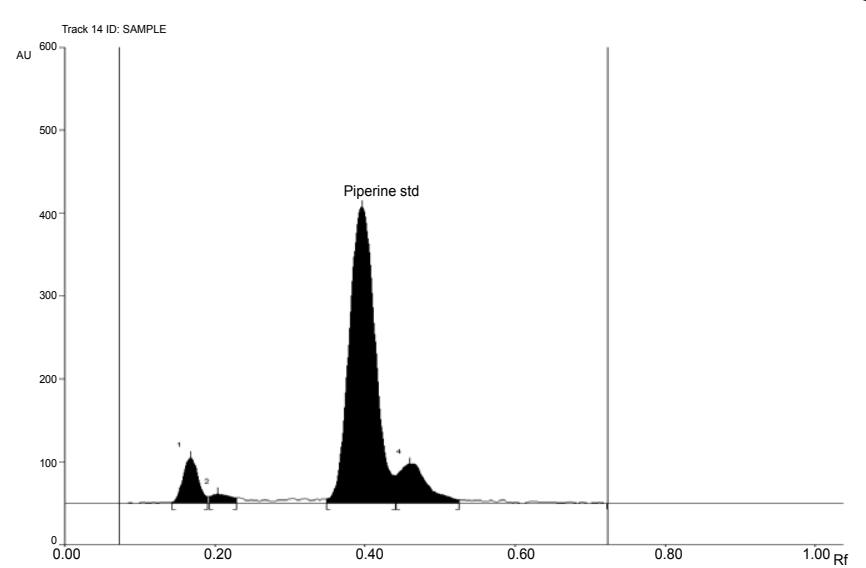

Figure 4: HPTLC Chromatogram of silver nanoparticles synthesized using Piper nigrumextract.

Production of cytokines are key events in the regulation of an inflammatory response and recent attention has been focussed on the effect of the synthesised nanoparticles as selective cytokine inhibitory agents. The cytokine inhibitory effect of the synthesized silver nanoparticles was ascertained using in vitro assays for TNFa, IL- $1 \beta$ and IL-6. TNF $\alpha$ inhibition was measured using RT PCR and the synthesized silver nanoparticles showed potent inhibition at lower concentrations, but higher concentrations showed decreased inhibition. Similar effects were also obtained with the Piper nigrum extract. This decrease in inhibitory effect with increase in concentration may be due to the priming effect of Piper nigrum extract. It can be seen that for IL- $1 \beta$ and IL- 6 assay, the synthesized silver nanoparticles showed potent inhibition even at lower concentrations when compared to the commercial silver nanoparticles and the plant extract alone. This can be explained by the synergistic anti inflammatory action of silver and

\section{Human IL-1 $\beta$ mRNA expression}

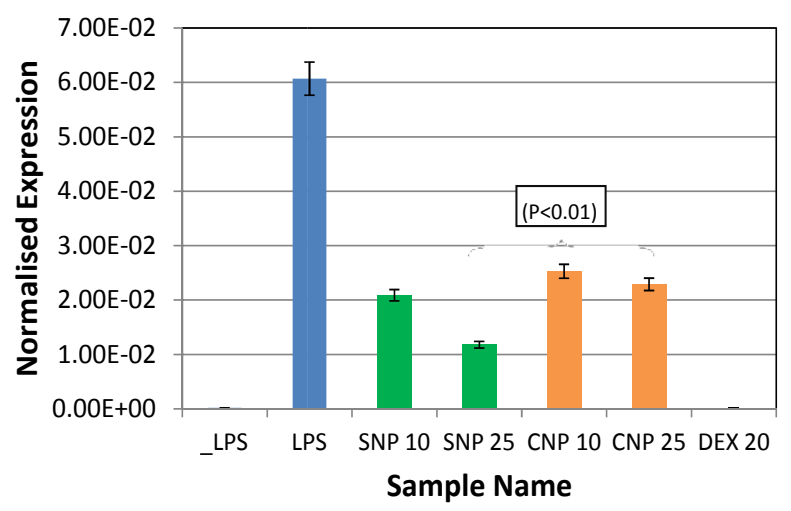

Figure 5: Inhibition of IL-1 $\beta$ mRNA expression by synthesized silver Nanoparticles Commercial silver Nanoparticles (CNP) in PBMC cells.

various phytochemicals present in Piper nigrum extract.

The effect of piperine (a major phytochemical present in Piper nigrum) on the pro inflammatory cytokines has also been studied extensively. Piperine inhibited the expression of IL6 and Matrix Metalloproteinase (MMP) 13 and reduced the production of PGE2 in a dose dependant manner at concentrations of 10 to $100 \mu \mathrm{g} / \mathrm{ml}$ [15]. In a study by Chuchawankul et al., Piperine also inhibited IL-2 and Interferon (IFN) $-\gamma$ production by activated PBMCs in a dosedependent manner [16].

The effects of silver nanoparticles on the production of cytokines by PBMC stimulated by phytohaemagglutinin (PHA) were studied by Shin et al. [16]. Silver nanoparticles strongly inhibited cytokine production, of INF- $\gamma$, IL-6, IL- 8, IL-11, TNF- $\alpha$ and more weakly IL-5. The mechanism of anti inflammatory effect is due to cellular 
Citation: Aparna Mani KM, Seethalakshmi S, Gopal V (2015) Evaluation of In-vitro Anti-Inflammatory Activity of Silver Nanoparticles Synthesised using Piper Nigrum Extract. J Nanomed Nanotechnol 6: 268. doi:10.4172/2157-7439.1000268

Page 5 of 5

\section{Human TNF $\alpha$ mRNA expression}

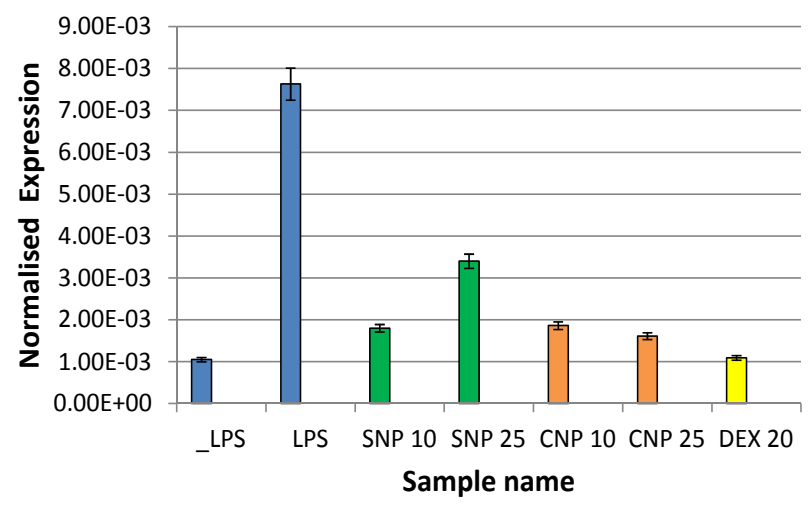

Figure 6: Inhibition of TNFa mRNA expression by Synthesized silver Nanoparticles (SNP) and Commercial silver Nanoparticles (CNP) in PBMC cells.

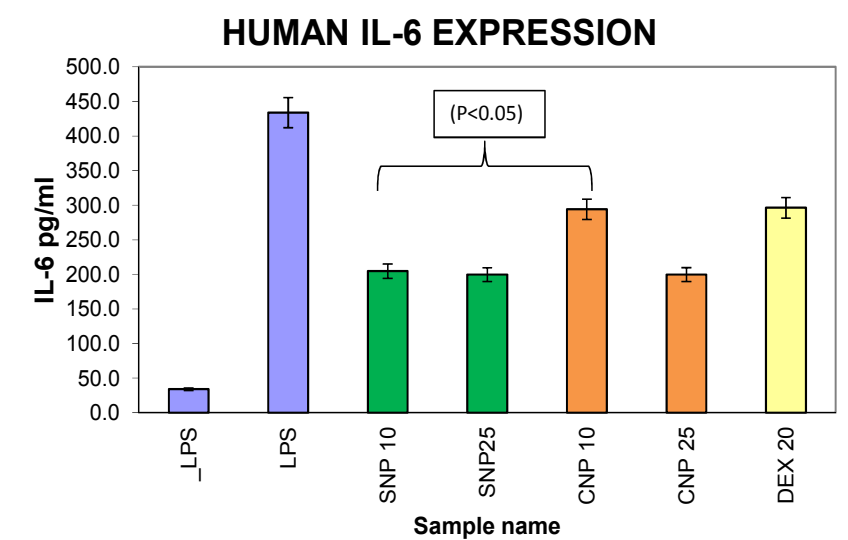

Figure 7: Inhibition of IL-6 expression by synthesized silver Nanoparticles (SNP) and Commercial silver Nanoparticles (CNP) in PBMC cells.

cytotoxicity a higher doses and also nano-silver was found to strongly effect the production of Th1cells that secrete the inflammatory cytokines IL-2, INF- $\gamma$ which are involved in cellular immunity, and in chronic inflammatory disorders [17].

\section{Conclusion}

This study has revealed that the green synthesis of silver nanoparticles using Piper nigrum extract has resulted in the formation of biologically active silver nanoparticles of size 40-100 nm. The synthesised silver nanoparticles are capped by the alkaloids of Piper nigrum especially Piperine and show significant anti inflammatory effects. In conclusion combining the benefits of phytomedicine with nanomedicne can result in the formation of more efficient silver nanoparticles with minimal toxic effects. This finding suggests a novel pharmacological rationale for the treatment of various inflammatory disorders.

\section{Acknowledgement}

The authors are thankful to The Indian Council for Medical Research (ICMR), New Delhi for providing financial support in the form of TSS fellowship (No. PhD (Integrated)/-17-F.T./2011)

\section{References}

1. Ricciotti E, FitzGerald GA (2011) Prostaglandins and inflammation. Arterioscler Thromb Vasc Biol 31: 986-1000.
2. Recio MC, Andujar I, Rios JL (2012) Anti-inflammatory agents from plants: progress and potential. Curr Med Chem 19: 2088-2103.

3. Anilkumar M (2010) Ethnomedicinal plants as antiinflammatory and analgesic agents. Ethnomedicine: A Source of Complementary Therapeutics: 267-293.

4. Rates SM (2001) Plants as source of drugs. Toxicon 39: 603-613.

5. Galdiero S, Falanga A, Vitiello M, Cantisani M, Marra V, et al. (2011) Silver Nanoparticles as Potential Antiviral Agents. Molecules 16: 8894-8918.

6. Wong KK, Cheung SO, Huang L, Niu J, Tao C, et al. (2009) Further evidence of the anti-inflammatory effects of silver nanoparticles. ChemMedChem 4: 11291135 .

7. Jeyaraj M, Sathishkumar G, Sivanandhan G, MubarakAli D, Rajesh M, et al (2013) Biogenic silver nanoparticles for cancer treatment: an experimental report. Colloids Surf B Biointerfaces 106: 86-92.

8. Fayaz AM, Balaji K, Girilal M, Yadav R, Thangavelu P, et al. (2010) Biogenic synthesis of silver nanoparticles and their synergistic effect with antibiotics: a study against gram-positive and gram-negative bacteria. Nanomedicine: Nanotechnology, Biology and Medicine 6: 103-109.

9. Ahmad N, Fazal H, Haider B, Farooq S, Ali M, et al. (2012) Biological role of Piper nigrum L. (Black pepper): A review. Asian Pacific Journal of Tropical Biomedicine 2: S1945-S1953.

10. Srinivasan K (2007) Black pepper and its pungent principle-piperine: a review of diverse physiological effects. Crit Rev Food Sci Nutr 47: 735-748.

11. Gülçin I (2005) The antioxidant and radical scavenging activities of black pepper (Piper nigrum) seeds. Int J Food Sci Nutr 56: 491-499.

12. Patil UK, Singh A, Chakraborty AK (2011) Role of Piperine As A Bioavailability Enhancer. International Journal of Recent Advances in Pharmaceutical Research 4: 16-23

13. Mani A, Seethalakshmi S, Gopal V (2012) Bio-Mimetic Synthesis of Silver Nanoparticles and Evaluation of its Free Radical Scavenging Activity. International Journal of Biological \& Pharmaceutical Research 3: 631-633.

14. Jyoti A, Dubey M, Kothari N (2012) Cytokines Induced Neutrophil Extracellular Traps Formation: Implication for the Inflammatory Disease Condition. PLoS One 7: e48111.

15. Bang JS, Oh da H, Choi HM, Sur BJ, Lim SJ, et al. (2009) Anti-inflammatory and antiarthritic effects of piperine in human interleukin 1beta-stimulated fibroblastlike synoviocytes and in rat arthritis models. Arthritis Res Ther 11: R49.

16. Shin SH, Ye MK, Kim HS, Kang HS (2007) The effects of nano-silver on the proliferation and cytokine expression by peripheral blood mononuclear cells. In Immunopharmacol 7: 1813-1818.

17. Chuchawankul S, Khorana N, Poovorawan Y (2012) Piperine inhibits cytokine production by human peripheral blood mononuclear cells. Genet Mol Res 11 : 617-627. 